\title{
Caracterización de carbones para la inyección por toberas en el horno alto
}

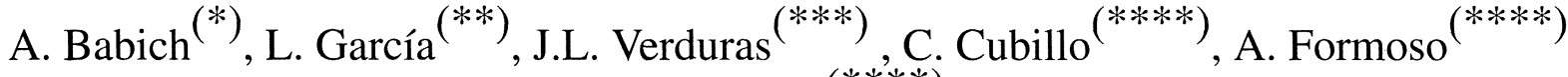 \\ y A. Cores $(* * * *)$
}

Resumen La eficacia de la operación del horno alto con inyección de carbón pulverizado (ICP) por toberas, está determinada por la composición y propiedades de los carbones utilizados y por la calidad de la carga férrea y del coque. Se realiza el estudio en termobalanza de carbones destinados a la inyección por toberas y se determinan las temperaturas de reblandecimiento y fusión de la ceniza de estos carbones. Se estima el comportamiento de los carbones y su influencia en la operación del horno alto.

Palabras clave: Carbón pulverizado. Ceniza de carbón. Inyección por toberas. Propiedades de la escoria. Horno alto.

\section{Coals characterization for blast furnace tuyeres inyection}

Abstract The efficiency of blast furnace operation with pulverized coal injection (PCI) by tuyeres is determined by the composition and properties of the used coals and by the quality of the ferrous burden and coke. A study in thermobalance of coals to be injected by tuyeres is carried out, and the softening and melting temperatures of coals ash are determined. The coal performance and its influence in the blast furnace operation is estimated

Keywords: Pulverized coal. Coal ash. Tuyeres injection. Slag properties. Blast furnace.

\section{INTRODUCCIÓN}

En los últimos años se han realizado trabajos sobre el proceso de combustión de carbones y de la tecnología de operación del horno alto con inyección de carbón pulverizado (ICP) por toberas. Las particularidades de estos trabajos son las siguientes:

a) Estudios de laboratorio de carbones destinados a la utilización en el horno alto, relacionados no sólo con la combustión, sino también con la descomposición.

b) Las investigaciones de combustión se realizan para carbones destinados a la preparación del CP y para el coque. Esto permite hacer un análisis comparativo de los procesos.

(*) Univ. Estatal Tecnológica de Donetsk. Donetsk (Ucrania).

(**) Unión de Empresas de Recuperación de Materias Primas. La Habana (Cuba).

(***) Aceralia Corporación Siderúrgica. Gijón (España).

(****) Centro Nacional de Investigaciones Metalúrgicas, CENIM (CSIC), Avda. de Gregorio del Amo, 8. 28040Madrid (España).
Para operar el horno alto con ICP es necesario utilizar una carga férrea y coque de alta calidad con el fin de mantener las condiciones gaseodinámicas de fusión, y una temperatura elevada del viento que permita garantizar el potencial térmico del crisol y una gasificación completa de las partículas de carbón. La experiencia de operación de muchos hornos en Europa y Japón confirma lo anterior ( 1 y 2).

En el presente trabajo se realizan estudios termográficos de carbones que se inyectan por toberas en los hornos altos de Aceralia Corporación Siderúrgica, instalados en Gijón, y en hornos de la factoría siderúrgica de Donetsk, en Ucrania.

\section{ENSAYOS DE LABORATORIO DE DESCOMPOSICIÓN Y COMBUSTIÓN DE CARBONES}

La composición química de los carbones y el comportamiento durante la combustión y oxidación influye de forma notable en la eficacia de la operación del horno alto con ICP. 
Se han realizado estudios teóricos sobre las variaciones de los índices técnicos y económicos de la fusión al inyectar al horno diferentes clases de carbones (3 y 4). También se ha analizado la operación del horno con el empleo de diferentes carbones (5).

En este trabajo se realizan estudios termográficos de diferentes carbones. En la tabla I se incluye el análisis químico y granulométrico de los carbones.

\subsection{Descomposición de carbones}

Para estudiar el mecanismo y la cinética de los procesos de transformación de los carbones, se realizan investigaciones termográficas en la termobalanza SETERAN, que describe las variaciones respecto al tiempo de los factores: temperatura, masa, velocidad de variación de masa y entalpia de los carbones (análisis termogravimétrico y termicodiferencial). Los resultados obtenidos se ofrecen en la tabla II.

En atmósfera inerte, entre $80-120^{\circ} \mathrm{C}$, se produce la eliminación del agua de absorción (humedad). Entre $120-420^{\circ} \mathrm{C}$, se desprende una pequeña cantidad de gases, principalmente $\mathrm{CO}$ y $\mathrm{CO}_{2}$, y comienza la disociación de la materia orgánica de los carbones. En el intervalo de $420-600{ }^{\circ} \mathrm{C}$, se produce un desprendimiento máximo de materias volátiles, contenidas en los hidrocarburos pesados, que poseen una relación $\mathrm{C}: \mathrm{H}$ bastante alta, tales como: metano, alquitranes, hidrocarburos nafténicos y aromáticos, fenoles pesados y algo de hidrógeno; en este intervalo se inicia una profunda disociación de los carbones y "se engendran" centros cristalizados de carbono primario. Entre $600-820^{\circ} \mathrm{C}$ se desprende la mayor cantidad de materiales volátiles (principalmente $\mathrm{CH}_{4}, \mathrm{CO} y$, sobre todo, $\mathrm{H}_{2}$ y también algo de $\mathrm{N}_{2}$ en forma de amoníaco y piridina) y prácticamente ya no hay presencia de alquitranes; en esta etapa, se forman algunos bloques de carbono. A $820-1.000{ }^{\circ} \mathrm{C}$, se desprenden las restantes materias volátiles, como hidrógeno, piridina y quinoleina, y también ocurren los procesos de asociación de bloques aislados en grandes sistemas de carbono.

En general, el proceso de descomposición de los carbones termina a $820^{\circ} \mathrm{C}$. La máxima intensidad de desprendimiento de volátiles, durante la descomposición, se observa a $420-600{ }^{\circ} \mathrm{C}$. En este intervalo se pierde la mitad de la masa total.

\subsection{Combustión de carbones}

El estudio de la combustión de los carbones se realiza en atmósfera de aire. El análisis de los derivatogramas permite dividir el proceso de combustión en las etapas siguientes: En la primera etapa se desprende la humedad contenida en las muestras. Este proceso termina a $150-200{ }^{\circ} \mathrm{C}$, en dependencia de la velocidad de calentamiento y del tipo de carbón. En la segunda etapa ocurre la quimioadsorción, relacionada con los procesos de adsorción (4). La tercera etapa se concluye con la liberación de los volátiles y se realiza a $350-600{ }^{\circ} \mathrm{C}$. En la última etapa se combustiona el coque residual (char) del carbón. La ignición ocurre entre $570-620^{\circ} \mathrm{C}$. El proceso de combustión concluye a $730-880^{\circ} \mathrm{C}$. Posteriormente, se observa una pequeña disminución de masa relacionada con la combustión del carbono

TABLA I.- Composición y granulometría de los carbones, \% en masa

TABLE I.- Composition and granulometry of coals, $\%$ in mass

\begin{tabular}{|l|c|c|c|c|r|r|}
\hline & Logan-K & Welch & Donbass-1 & Donbass-2 & Guasare & Prodeco \\
\hline Análisis inmediato & & & & & & \\
Carbono fijo & 58,6 & 75,6 & 76,9 & 75,68 & 57,22 & 52,65 \\
Volátiles & 34,1 & 16,7 & 13,0 & 13,09 & 37,35 & 38,15 \\
Ceniza & 7,3 & 7,7 & 8,3 & 11,23 & 5,43 & 9,21 \\
Humedad & 1,4 & 0,8 & 0,5 & 1,12 & 2,08 & 4,99 \\
Análisis elemental & & & & & & \\
Carbono & 80,5 & 85,4 & 83,7 & 79,43 & 79,57 & 73,74 \\
Hidrógeno & 5,1 & 3,9 & 3,40 & 3,72 & 5,26 & 5,81 \\
Nitrógeno & 1,6 & 1,1 & 1,50 & 1,67 & 1,48 & 1,36 \\
Azufre & 0,75 & 0,72 & 1,80 & 1,14 & 0,72 & 0,78 \\
Oxígeno & 4,75 & 1,2 & 1,30 & 2,81 & 7,54 & 9,01 \\
Granulometría, $\mu \mathrm{m}$ & & & & & & \\
>105 & 12,1 & 13,8 & 0,04 & 8,6 & 9,79 & 15,76 \\
105-88 & 11,2 & 11,0 & 0,41 & 9,6 & 11,65 & 13,60 \\
$\quad 88-53$ & 21,1 & 17,0 & 7,84 & 9,4 & 19,57 & 20,80 \\
$<53$ & 54,8 & 58,2 & 92,07 & 72,4 & 59,99 & 49,30 \\
\hline
\end{tabular}


TABLA II.- Pérdidas de masa (\%) de los carbones en los ensayos en termobalanza

TABLE II.-Lost of mass (\%) of coals in thermobalance tests

\begin{tabular}{|c|c|c|c|c|c|c|c|c|}
\hline \multirow[t]{2}{*}{ Carbón } & \multirow[t]{2}{*}{ Atmósfera } & \multirow{2}{*}{$\begin{array}{l}\text { Velocidad de } \\
\text { calentamiento, } \\
{ }^{\circ} \mathrm{C} / \mathrm{min}\end{array}$} & \multicolumn{5}{|c|}{ Intervalos de temperaturas, ${ }^{\circ} \mathrm{C}$} & \multirow{2}{*}{$\begin{array}{l}\text { Pérdida } \\
\text { de masa } \\
\text { total, \% }\end{array}$} \\
\hline & & & $80-120$ & $120-420$ & $420-600$ & $600-820$ & $820-1000$ & \\
\hline \multirow{4}{*}{ LOGAN-K } & Aire & 1000 & 1,66 & 3,32 & 49,34 & 30,49 & 14,45 & 99,26 \\
\hline & & 600 & 2,16 & 5,56 & 67,04 & 22,86 & - & 97,62 \\
\hline & $\mathrm{N}_{2}$ & 1000 & 2,17 & 4,83 & 20,77 & 9,66 & 3,62 & 41,05 \\
\hline & & 600 & 2,12 & 5,43 & 21,24 & 8,50 & 2,36 & 39,65 \\
\hline \multirow{4}{*}{ WELCH } & Aire & 1000 & 1,26 & 4,41 & 35,62 & 31,52 & 26,16 & 98,97 \\
\hline & & 600 & 1,12 & 8,23 & 47,54 & 40,05 & - & 96,94 \\
\hline & $\mathrm{N}_{2}$ & 1000 & 1,31 & 3,06 & 10,49 & 7,43 & 1,31 & 23,60 \\
\hline & & 600 & 1,22 & 2,43 & 9,32 & 6,49 & 3,65 & 23,11 \\
\hline \multirow{4}{*}{ DONBASS } & Aire & 1000 & 1,53 & 3,84 & 46,42 & 26,09 & 18,03 & 95,91 \\
\hline & & 600 & 1,40 & 3,50 & 76,04 & 12,62 & - & 93,56 \\
\hline & $\mathrm{N}_{2}$ & 1000 & 0,92 & 2,15 & 8,29 & 7,06 & 5,53 & 23,95 \\
\hline & & 600 & 0,97 & 1,94 & 8,49 & 3,39 & 3,39 & 18,18 \\
\hline \multirow{4}{*}{ GUASARE } & Aire & 1000 & 1,78 & 3,19 & 47,85 & 29,44 & 17,66 & 99,92 \\
\hline & & 600 & 1,67 & 6,96 & 68,77 & 21,26 & - & 98,66 \\
\hline & $\mathrm{N}_{2}$ & 1000 & 2,26 & 3,96 & 22,63 & 15,00 & 3,38 & 47,23 \\
\hline & & 600 & 2,24 & 4,75 & 19,86 & 15,38 & 3,35 & 45,58 \\
\hline \multirow{4}{*}{ PRODECO } & Aire & 1000 & 6,05 & 6,05 & 41,29 & 25,88 & 19,27 & 98,54 \\
\hline & & 600 & 3,89 & 9,90 & 68,22 & 15,55 & - & 97,56 \\
\hline & $\mathrm{N}_{2}$ & 1000 & 5,78 & 5,13 & 20,54 & 15,08 & 6,10 & 52,36 \\
\hline & & 600 & 4,94 & 6,26 & 19,44 & 19,77 & 5,27 & 55,68 \\
\hline
\end{tabular}

enmascarado en la ceniza. $\mathrm{Al}$ aumentar la velocidad de calentamiento de 600 a $1000{ }^{\circ} \mathrm{C} / \mathrm{h}$, la combustión del carbón es más rápida y completa. La combustión del char, en el intervalo de altas temperaturas $\left(600-1.000{ }^{\circ} \mathrm{C}\right)$, es más intensa para una velocidad de calentamiento elevada.

La aptitud a la combustión de los carbones, con la excepción del Donbass, se estudió en la planta piloto del CENIM, equipada con una cámara de combustión que simula la zona de toberas-raceway (6 y 7).

\section{INFLUENCIA DE LA CENIZA DE LOS CARBONES EN LA OPERACIÓN DEL HORNO ALTO}

La composición y propiedades de la ceniza de los carbones inyectados por toberas en el horno ejercen una influencia notable en la escoria, en la combustión en las toberas y en la distribución uniforme del viento en la periferia del horno en la zona de toberas. En la tabla III se incluye la composición química de la ceniza de los carbones.

\subsection{Consumo de fundente y volumen de escoria}

Por cuanto la ceniza de todos los carbones es ácida, la ICP requerirá de algún consumo de fun- dente para la formación de escoria a partir de la ceniza. Como resultado, aumenta el volumen de escoria. El consumo de fundente $(\mathrm{kg} / \mathrm{kg}$ de $\mathrm{CP})$ para la escorificación de la ceniza se determina mediante la ecuación:

$$
f=\left(\mathrm{SiO}_{2} \cdot \mathrm{B}-\mathrm{CaO}\right) A_{\mathrm{CP}} / 100 \cdot \mathrm{CaO}_{\mathrm{f}}
$$

donde,

$\mathrm{SiO}_{2}$ y $\mathrm{CaO}=$ contenido de $\mathrm{SiO}_{2}$ y $\mathrm{CaO}$ en la ceniza del CP, \%;

$\mathrm{CaO}_{\mathrm{f}}=$ contenido de $\mathrm{CaO}$ en el fundente, $\%$;

$\mathrm{B} \quad=$ índice de basicidad de la escoria $\left(\mathrm{CaO} / \mathrm{SiO}_{2}\right)$;

$\mathrm{A}_{\mathrm{CP}} \quad=$ contenido de ceniza en el CP, $\%$.

El volumen de escoria $(\mathrm{kg} / \mathrm{kg}$ de $\mathrm{CP})$ de la ceniza del CP y el correspondiente consumo complementario de fundente se determina por la ecuación:

$$
\begin{gathered}
U_{\mathrm{CP}}=0,01\left[\mathrm{~A}_{\mathrm{CP}}+\mathrm{f}\left(\mathrm{CaO}+\mathrm{SiO}_{2}+\mathrm{MgO}+\right.\right. \\
\left.\left.+\mathrm{Al}_{2} \mathrm{O}_{3}+\Omega \mathrm{MnO}\right)\right]
\end{gathered}
$$

donde,

$\mathrm{CaO}, \mathrm{SiO}_{2}, \mathrm{MgO}, \mathrm{Al}_{2} \mathrm{O}_{3}, \mathrm{MnO}=$ contenido de los respectivos componentes en el fundente, \%;

$\Omega=$ grado de transferencia del Mn a la escoria, unidades. 
TABLA III.- Composición química de la ceniza de los carbones, \% en masa

TABLE III.-Chemical composition of coals' ash, \% in mass

\begin{tabular}{|l|c|c|c|c|c|c|}
\hline & Logan- $\mathrm{K}$ & Welch & Donbass- 1 & Donbass-2 & Guasare & Prodeco \\
\hline $\mathrm{SiO}_{2}$ & 53,55 & 50,50 & 40,80 & 51,42 & 55,54 & 63,65 \\
$\mathrm{Al}_{2} \mathrm{O}_{3}$ & 29,85 & 27,64 & 23,60 & 29,46 & 23,24 & 17,37 \\
$\mathrm{Fe}_{2} \mathrm{O}_{3}$ & 7,56 & 10,50 & 19,20 & 9,00 & 10,95 & 5,99 \\
$\mathrm{CaO}$ & 2,13 & 2,78 & 3,34 & 2,19 & 0,92 & 4,73 \\
$\mathrm{MgO}$ & 1,05 & 1,17 & 1,15 & 1,16 & 1,10 & 3,20 \\
$\mathrm{TiO}_{2}$ & 1,99 & 1,54 & - & 1,06 & 1,43 & 0,69 \\
$\mathrm{Mn}_{3} \mathrm{O}_{4}$ & 0,04 & 0,06 & 0,26 & 0,06 & 1,36 & 0,09 \\
$\mathrm{P}_{2} \mathrm{O}_{5}$ & 0,34 & 0,44 & 0,60 & 0,51 & 0,22 & 0,30 \\
$\mathrm{SO}_{3}$ & 0,167 & 1,15 & - & 0,30 & 0,036 & 1,23 \\
$\mathrm{Na}_{2} \mathrm{O}$ & 0,20 & 0,79 & 0,12 & 0,96 & 0,44 & 0,97 \\
$\mathrm{~K}_{2} \mathrm{O}$ & 1,97 & 2,04 & 0,40 & 3,36 & 2,38 & 1,00 \\
\hline
\end{tabular}

Se ha calculado para los carbones el consumo de caliza, utilizada como fundente, para el paso a la escoria de la ceniza del carbón y el volumen complementario de escoria a partir de la ceniza. Como caudal de ICP se considera $150 \mathrm{~kg}$ de $\mathrm{CP} / \mathrm{ta}$. El resultado se muestra en la tabla IV.

Por consiguiente, la cantidad y composición de la ceniza del carbón influye notablemente en el volumen e índice de basicidad de la escoria. Para conservar la capacidad de desulfuración y otras características de la escoria, es necesario aumentar la cantidad de fundente o acometer otras medidas especiales.

\subsection{Reblandecimiento y fusión de la ceniza}

Las propiedades físicas de la ceniza del CP influyen en las condiciones de trabajo de las toberas y en las zonas de oxidación. Esto se relaciona con propiedades de la ceniza, tales como viscosidad, y las temperaturas de reblandecimiento y fusión. Las influencias señaladas se explican por lo siguiente:

Durante la combustión de las partículas de los residuos de carbón (char), la temperatura de las partículas crece en el canal del dispositivo de tobe- ras, a la salida de la lanza del CP. Si la ceniza del $\mathrm{CP}$ tiene una temperatura de reblandecimiento comparable a la temperatura de toberas, es posible la fusión de la ceniza del carbón con el consiguiente peligro de adherencia en la superficie interna de las toberas, lo que puede ocasionar el atascamiento de las toberas, y por tanto empeorar la operación del horno.

Las temperaturas de reblandecimiento y fusión de la ceniza de los carbones se estudiaron en un equipo LECO que alcanza $1.650^{\circ} \mathrm{C}$. Los resultados se muestran en la tabla V.

El carbón Guasare, que actualmente se utiliza para la ICP en el HA-B de Aceralia, tiene una ceniza cuyo reblandecimiento ocurre a $1.407^{\circ} \mathrm{C}$ y la fusión a $1.494^{\circ} \mathrm{C}$.

Las características de reblandecimiento y fusión de las cenizas de los carbones Logan-K y Welch son semejantes a las de la ceniza del carbón Guasare. Desde el punto de vista de la influencia de la ceniza en las toberas y del régimen de escoria, se pueden recomendar estos carbones para su inyección en los hornos de Gijón.

El carbón Prodeco tiene temperaturas de reblandecimiento y fusión de la ceniza más bajas. Cuando se introduce en el viento a $1.200{ }^{\circ} \mathrm{C}$, la temperatura

TABLA IV.- Consumo de fundente y volumen de escoria

TABLE IV.- Flux consumption and slag volume

\begin{tabular}{|l|c|c|c|c|c|c|}
\hline Índices & Logan-K & Welch & Donbass-1 & Donbass-2 & Guasare & Prodeco \\
\hline $\begin{array}{l}\text { Consumo de caliza } \\
\mathrm{kg} / \mathrm{kgCP}\end{array}$ & 0,080 & 0,079 & 0,070 & 0,127 & 0,063 & 0,117 \\
$\begin{array}{l}\text { Consumo de caliza } \\
\text { para } 150 \mathrm{kgCP} / \mathrm{ta}\end{array}$ & 12,06 & 11,83 & 10,50 & 19,05 & 9,50 & 17,53 \\
$\begin{array}{l}\text { Volumen de escoria } \\
\mathrm{kg} / \mathrm{kg} \mathrm{CP}\end{array}$ & 0,119 & 0,122 & 0,123 & 0,185 & 0,090 & 0,159 \\
$\begin{array}{l}\text { Volumen de escoria } \\
\text { para } 150 \mathrm{kgCP} / \mathrm{ta}\end{array}$ & 18,81 & 18,32 & 18,45 & 27,73 & 13,54 & 23,84 \\
\hline
\end{tabular}


TABLA V.- Temperaturas de reblandecimiento y fusión de la ceniza de los carbones TABLE V.- Softening and melting temperatures of coals' ash

\begin{tabular}{|l|c|c|c|c|}
\hline \multirow{2}{*}{ Carbón } & \multicolumn{4}{|c|}{ Valor medio de puntos representativos, ${ }^{\circ} \mathrm{C}$} \\
\cline { 2 - 5 } & IT & ST & HT & FT \\
\hline Logan-K & 1.300 & 1.455 & 1.489 & 1.491 \\
Welch & 1.324 & 1.447 & 1.464 & 1.468 \\
Donbass-3 & 1.269 & 1.468 & 1.493 & 1.497 \\
Guasare & 1.354 & 1.407 & 1.431 & 1.494 \\
Prodeco & 1.254 & 1.269 & 1.295 & 1.384 \\
\hline
\end{tabular}

IT: temperatura de inicio de deformación

ST: temperatura de reblandecimiento

HT: temperatura de formación de la semiesfera

FT: temperatura de fusión

del medio gaseoso puede sobrepasar 1.300-1.380 ${ }^{\circ} \mathrm{C}$. En este caso, existe peligro de adherencia de las gotas de ceniza fundidas en la superficie interna de las toberas. El contenido elevado de humedad de este carbón $(5 \%)$ requiere de mayor energía en el secado con el fin de garantizar condiciones favorables para el transporte neumático.

Los factores expuestos señalan que la utilización del carbón Prodeco puede ocasionar problemas en la operación del horno y en las instalaciones de preparación e inyección del CP.

\section{CONCLUSIONES}

Durante la descomposición de carbones, la pérdida de masa es proporcional al contenido de materias volátiles. La disociación de carbones finaliza a $800-820^{\circ} \mathrm{C}$ y el desprendimiento más intenso de volátiles ocurre a $420-600^{\circ} \mathrm{C}$.

La combustión de carbones con el oxígeno del aire se desarrolla en cuatro etapas. Durante la combustión del coque, no existe la etapa de quimiadsorción y el desprendimiento de volátiles no se acompaña con efecto exotérmico. La ignición del coque ocurre a temperatura más alta que en los carbones.

La composición y propiedades de los carbones Logan-K, Welch, Donbass y Guasare permiten que se puedan inyectar en los hornos altos de Aceralia Corporación Siderúrgica, instalados en Gijón. El carbón Prodeco puede causar problemas en la operación del horno.

El estudio de las escorias recogidas en el horno alto que opera con caudal elevado de ICP, confirma que poseen buena fluidez y que son de fácil fusión. Esto hace posible una operación normal del horno con caudal elevado de inyección, incluso en condiciones poco satisfactorias de calidad de carga.

\section{Agradecimiento}

Los autores agradecen a la Comunidad Europea (ECSC 7210-AA/938) y Aceralia Corporación Siderúrgica el apoyo económico a este Proyecto.

\section{REFERENCIAS}

(1) Peters, K.H., Mohnkern, H. y Lüngen, H.B. Proc.1st Int. Congr. on Science and Technology of Ironmaking. ISIJ, Tokyo, 1994: 493-499

(2) Steiler, J.M., Dufour, A., Libralesso, J.M., Helleisen, M. y Harting, W. Rev. Metall. CIT, (10/11), 1995: 1.207-1.223.

(3) Babich, A., Yaroshevskit, S., Garcia, L., Formoso, A., Cores, A., Isidro, A. y Ferreira, S. Rev. Metal. Madrid, 32 (2), 1996: 103-116.

(4) Babich, A.I., YaroshevskiI, S.L. y Tereshenko, I.P. Intensificación de la utilización del carbón pulverizado en el horno alto. Kiev, "Ed. Tejnika", 1993: 200 pag., en ruso.

(5) Peters, K.H., Peters, M. y Korthas, B. Metall. Tech. Int. (6), 1990: 32-35.

(6) Isidro, A., Formoso, A., Pis, J.J., Ferreira, S., Fuente, E., Rivas, J.M., Garcia, L. y Cores, A. Proc. ViII Int. Conf. on Coal Science and Technology., Vol 1, Oviedo (España). Elsevier Science, 1995: 551-554.

(7) Babich, A., YaroshevskiI, S., Formoso, A., Isidro, A., Ferreira, S., Cores, A. y Garcia, L. ISIJ Int., 36(10), 1996: $1250-1258$. 